\title{
Review of the recent controlled experiments for study of local reconnection physics
}

\author{
Masaaki Yamada \\ Princeton Plasma Physics Laboratory, P.O. Box 451, Princeton, NJ 08543, U.S.A. \\ (Received June 26, 2000; Revised January 25, 2001; Accepted February 28, 2001)
}

\begin{abstract}
The present paper reviews the recent laboratory experiments on magnetic reconnection focussing on the local features of the reconnection region. It is very important to recognize that magnetohydrodynamics (MHD) often breaks down locally in the thin reconnection layer, while globally, the reconnecting plasma has large Lundquist number and is well approximated by MHD equations. Precise measurements of the neutral sheet profile can provide important clues to help understand the non-MHD physics mechanisms of reconnection. Thanks to significant progress in data acquisition technology, the detailed magnetic field structure of the neutral sheet has been measured in laboratory plasmas. Extensive data have been accumulated in highly conductive MHD plasmas with large Lundquist numbers $S=10-1000$. In this review we primarily focus on the physics data of the neutral sheet from the most recent laboratory experiments.
\end{abstract}

\section{Introduction}

Magnetic reconnection occurs where two conductive plasmas of different magnetic configuration meet. The reconnection region, which is often called a diffusion region or neutral sheet, is a focal point of MHD plasma phenomena. Figure 1 depicts a two-dimensional (2-D) schematic of the diffusion region together with actually measured magnetic field profiles in a laboratory plasma (Yamada et al., 1997a,b; Ji et al., 1998). Its treatment fundamentally invokes issues of non-linear MHD theories and often non-MHD physics on globally conductive plasmas with large Lundquist number $S$ [which is defined as the ratio of the magnetic diffusion time to the Alfven transit time]. This paper reviews results from the most recent experiments in which the magnetic reconnection region has been generated and studied in controlled laboratory settings.

In the past decades, research on the fundamental physics of the reconnection process and its hydromagnetic consequences has been largely theoretical (Priest and Forbes, 2000) and has been primarily based on steady-state 2-D magnetohydrodynamic (MHD) models. In the collisionless regime $\left(\lambda_{m f p} \gg\right.$ Neutral sheet width), these theories are expected to break down or require a significant modification. Although the Sweet-Parker $(1958,1957)$ and Petschek (1964) models are well known, the extensive literature describing these 2-D theoretical models thus remains to be verified in laboratory experiments or by space observations.

Laboratory experiments are very useful for understanding the fundamental physics of magnetic reconnection since they can provide well-correlated plasma parameters at multiple plasma locations simultaneously while satellites can only provide information from a single location at a given time in a space plasma. During the past decades, a significant

Copy right (C) The Society of Geomagnetism and Earth, Planetary and Space Sciences (SGEPSS); The Seismological Society of Japan; The Volcanological Society of Japan; The Geodetic Society of Japan; The Japanese Society for Planetary Sciences. progress in data processing technology has made it possible to acquire s large amount of data from the plasma interior within a short time. This has enabled us to measure the time evolution of the 2-D fine structure of the reconnecting region (neutral sheet) during the merging of two plasmas. Another important development is that in modern experiments the effects of the third vector component of merging field lines have been studied.

The fundamental physics of magnetic reconnection is studied through two different perspectives, (1) local and (2) global views. In local analysis it is assumed that reconnection mechanisms are determined primarily by local plasma parameters in the reconnecting region; the Sweet-Parker model and Petschek model use such an assumption. In this analysis, boundaries are not specified and external conditions are often translated to the plasma flow into the reconnection region. It has been observed, however, that reconnection is often influenced and determined by external boundary conditions and the initial 3-D global configuration or topology. It has been found that a large MHD instability caused by external conditions can invoke magnetic reconnection and can determine its rate. In actual reconnection phenomena the reconnection process is in general determined both by local plasma properties and global plasma characteristics.

It is very important to note that MHD often breaks down locally in the reconnection layer, while globally, the plasma has a large Lundquist number and is well approximated by MHD equations. This is true particularly when the thickness of the neutral sheet is comparable to the ion gyro-radius. Precise measurements of the neutral sheet profile can provide important clues to help understand the non-MHD physics mechanisms of reconnection. Recently the magnetic field structure of the neutral sheet has been measured in detail in laboratory plasmas. Extensive data have been accumulated in highly conductive MHD plasmas with large Lundquist numbers $S=10-1000$. In this review we put a primary 
(a)

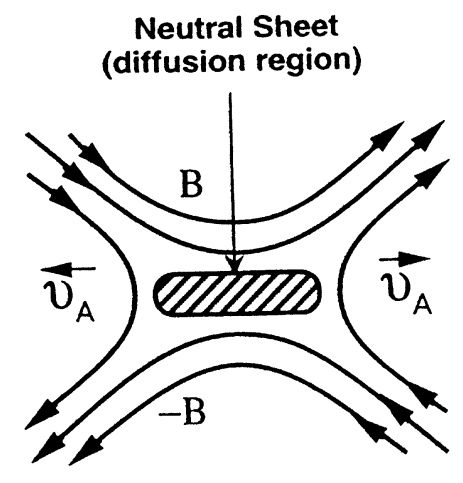

(b) Vector Plot

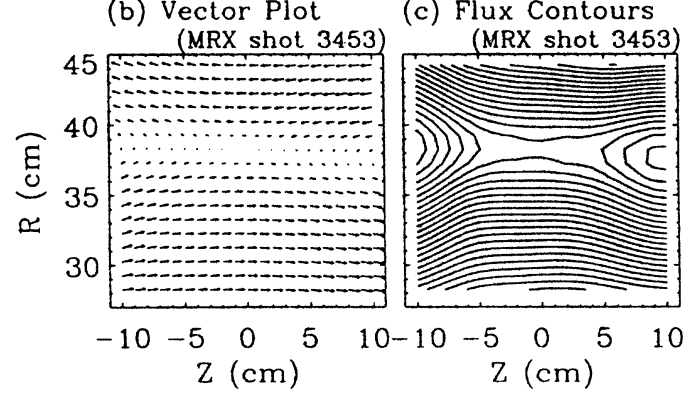

Fig. 1. 2-D view of merging field lines; schematic of the diffusion region together with actually measured magnetic field profiles in the MRX plasma (Yamada et al., 1997a,b; Ji et al., 1998). In the neutral sheet, conventional picture of MHD breaks down.

focus on the local studies of the reconnection region through data from the recent laboratory experiments.

\section{Recent Theoretical Development: From MHD to non-MHD Theories}

\subsection{Resistive MHD theories}

In the one-fluid MHD formulation, the motion of magnetic field lines in a plasma, can be described by an equation for magnetic field B by combining Maxwell equations and Ohm's law,

$$
\begin{aligned}
& \mathbf{E}+\mathbf{v} \times \mathbf{B}=\eta \mathbf{j} \\
& \frac{\partial \mathbf{B}}{\partial t}=\nabla \times(\mathbf{v} \times \mathbf{B})+\frac{\eta}{\mu_{0}} \nabla^{2} \mathbf{B} .
\end{aligned}
$$

where $\mathbf{E}$ is the electric field, $\mathbf{B}$ the magnetic field, $\mathbf{v}$ the particle velocity.

The magnitudes of the first and the second terms in the right hand side of Eq. (2) represent the inverse of the Alfven transit time, $\tau_{A}=L / v_{A}$ and the magnetic diffusion time, $\tau_{D}=\frac{\mu_{0} L^{2}}{\eta}$. The ratio $\frac{\tau_{D}}{\tau_{A}}$ is called Lundquist number, $S$. For MHD plasmas, $S$ is much larger than 1 and the second term is very small. When $\eta=0$, magnetic field lines move with the fluid as seen in Eq. (2). In resistive MHD plasmas, hydromagnetic flows can lead to neutral sheets where the plasma flow is reduced to near zero and the electric field $\mathbf{E}$ is balanced with $\eta \mathbf{j}$ in Eq. (1).

In this diffusion region, the resistivity becomes sufficiently large so that a magnetic field line can lose its original identity and reconnect to another field line. The topology of the magnetic configuration changes and large $\mathbf{j} \times \mathbf{B}$ hydromagnetic forces moves the plasma. Sweet and Parker developed a 2-D incompressible MHD model assuming that a local reconnection rate can be described by a steady state formulation $\frac{\partial \mathbf{B}}{\partial t}=0, \frac{\partial \mathbf{v}}{\partial t}=0$. Utilizing the continuity equation and pressure balance between the upstream $p \simeq \frac{B^{2}}{2 \mu_{0}}$ and the down stream $p \simeq \frac{\rho v^{2}}{2}$ regions, they derived a very simple formula for reconnection speed;

$$
\frac{V_{\mathrm{rec}}}{V_{A}}=\sqrt{\frac{\eta}{\mu_{0} L V_{A}}}=\frac{1}{\sqrt{S}} .
$$

Petschek proposed an alternative model (Petschek, 1964) which consists of a much smaller diffusion region and standing wave shock structure. The smaller size of the diffusion region allows a faster reconnection rate and this Petschek model has been favored over the Sweet-Parker model, because its faster predicted reconnection rate agrees better with space observations. However, the shock structure, an outcome of his model, has not been conclusively identified to date in laboratory experiments or space observations. In general most MHD theories of magnetic reconnection, which are based on steady-state 2-D models with uniform resistivity and laminar flows, do not predict the shock structure. But with the enhanced resistivity in the neutral region, some MHD theories lead to the Petschek type shock region (Sato and Hayashi, 1979). The effects of the axial component of magnetic field have been studied by many researchers.

\subsection{Non-MHD effects}

It has been known for decades that simple MHD theories do not properly describe magnetic reconnection phenomena. For example, it is well known that reconnection proceeds at a much faster rate than the classical rate in laboratory plasmas without the presence of shock structures. It has been conjectured that the cause of fast reconnection can be attributed to the so-called anomalous resistivity, when fluctuations are induced in the neutral sheet region. It has also been observed in MRX (Magnetic Reconnection Experiment, Yamada, 1997b) that the width of the reconnection region is on the order of ion skin depth as well as the ion gyro-radius and a simple MHD formulation is expected to break down in this regime.

Resolution of this dilemma can come from looking beyond the single fluid (MHD) approximation of plasmas, because the phenomena involved have shorter time scales than the collision time and have finer spatial scales than the mean free path. The relevant scales fall beyond the regime of validity of conventional MHD models. Recently much thought has been given to the 'generalized' Ohm's law where electrons and ions are separately treated to satisfy the equations of motion in the neutral sheet plasma. In this "E-MHD" region electrons but not ions are considered to be magnetized. The generalized Ohm's law describes force balance of an electron flow, namely,

$$
\mathbf{E}+\mathbf{v}_{e} \times \mathbf{B}=\eta_{s} \mathbf{j}+\frac{m_{e}}{e}\left(\frac{\partial \mathbf{v}_{e}}{\partial t}+\mathbf{v}_{e} \cdot \nabla \mathbf{v}_{e}\right)-\frac{\nabla \mathbf{P}_{e}}{e n} .
$$

In Eq. (4), all vectors include fluctuation components and $\eta_{s}$ denotes the classical Spitzer resistivity based on Coulomb collisions. In the neutral sheet we expect that the fluctuation 
components of $\mathbf{v}_{e} \times \mathbf{B}, \mathbf{v}_{e} \cdot \nabla \mathbf{v}_{e}$, and $\frac{\nabla \mathbf{P}_{e}}{e n}$ can make a significant contribution to the electrons' force balance and the energy dissipation rate, thus affecting the magnetic reconnection speed. If Eq. (4) is expressed as $\mathbf{E}=\eta_{s} j+\eta_{\text {anom }} j=\eta_{\text {eff }} j$, the crucial fluctuation terms $\frac{m_{e}}{e}\left(\frac{\partial \mathbf{v}_{e}}{\partial t}+\mathbf{v}_{e} \cdot \nabla \mathbf{v}_{e}\right)-\frac{\nabla \mathbf{P}_{e}}{e n}-\mathbf{v}_{e} \times \mathbf{B}$, are expected to be (non-linearly) proportional to the driving term, the electron current density, $j$, and they can be altogether expressed by $\eta_{\text {anom }} j$. It is therefore very important to investigate the nature of plasma fluctuations in the neutral sheet and many recent studies have been focussed on this issue.

A new type of realistic simulations using powerful computational resources are currently in progress (Drake et al., 1997; Biskamp et al., 1995; Horiuchi and Sato, 1997; Shay et al., 1998). Many of the recent simulations have shown that fast reconnection can occur in the collisionless neutral sheet. At present, there is no consensus as to the physics of the observed enhanced resistivity since there are so many different levels of approximations in the numerical models (i.e., single-fluid, two-fluid, fluid-particle hybrid, full particle simulations and 2-D or 3-D geometries) and so many candidate phenomena (ion acoustic waves, lower-hybrid waves, whistler waves etc.) to explain the observed enhanced resistivity. Thus it is very important to study reconnection in a laboratory setting in which important characteristics can be measured in situ and compared with numerical simulation results as well as with analytical theories.

\section{Neutral Sheet Profiles Measured in Early Lab- oratory Experiments}

The majority of early laboratory experiments on magnetic reconnection were carried out in 'pinch' plasmas, fast high-density pulsed plasma discharges of short duration of a few micro-seconds (Baum and Bratenahl, 1980; Syrovatskii, 1981; Ohyabu et al., 1974; Syrovatskii et al., 1973; Frank et al., 1974). In these experiments it was difficult to diagnose the reconnection region with good spatial and temporal resolution. In addition, the low Lundquist numbers $(S=1-10)$ attained in these transient experiments $(\partial B / \partial t \neq 0)$ made it difficult to compare the results with MHD theories which require $S \gg 1$ to be globally satisfied in the plasma. Despite these difficulties, the current density profiles of the neutral sheet were deduced from local magnetic probe measurements and density profiles were measured with a short time scale on the order of an Alfven transit time $\left(\tau_{A}<1\right.$ $\mu \mathrm{sec})$ by Syrovatskii (1981) and Frank et al. (1974). Figure 2(a) presents their experimental set up, where formation of a flat current sheet was made by a $z$-pinch discharge in which current was induced along the axis ( $z$ axis) of a straight cylinder. A linear pinch plasma was created by the breakdown of a neutral gas. The sheet was formed by an inductive pulsed electric field of 50-300 V applied between two electrodes separated by $40-60 \mathrm{~cm}$. Figure 2(b) shows the time evolution of reconnecting magnetic field profiles in the $y$ axis (based on their coordinate system which is not a conventional one), across the sheet. After magnetosonic waves converged a current sheet began to form in the vicinity of the null line being pinched along the $y$ axis and elongated along the $x$ axis. Figure 2(c) presents the electron density profile of the neutral sheet measured by laser interferome-

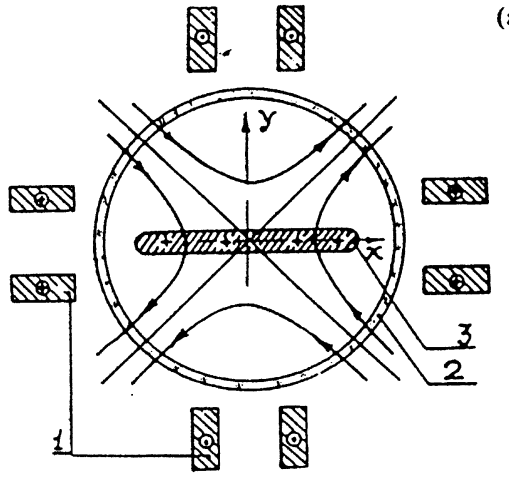

(a)
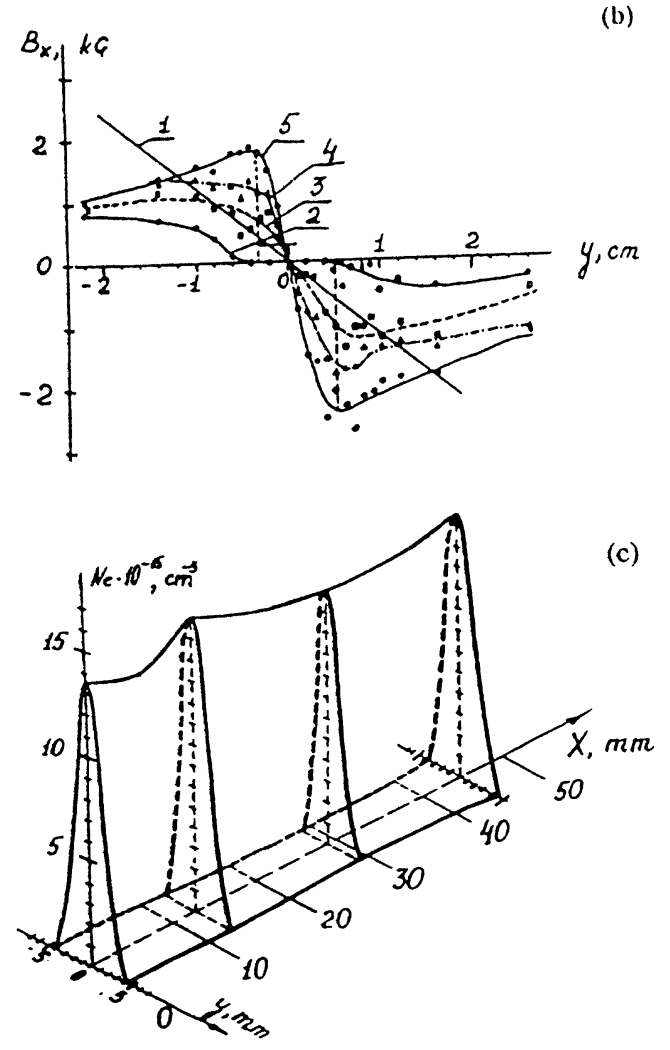

Fig. 2. A Set-up of $z$-pinch reconnection experiment by Syrovatskiii (1973), 1. Conductors, 2. Glass vacuum chamber, 3. current sheet; Vacuum filed lines are shown by arrows. b, Evolution of profile of reconnectiing field $B_{x}$ versus $y$ axis. 1 is for intial vacuum field, $2-5$ for $t=0.2,0.3,0.4$, $0.5 \mu \mathrm{sec}$. Gas fill pressure $\sim 0.06$ torr. c, Profile of elecron density in the neutral sheet measured by interferometry.

try. According to their conclusion the final thickness of the sheet appeared to be determined by the pressure balance between the reconnecting magnetic field and the plasma kinetic pressure (Syrovatskii, 1981). This force balance seemed to cause the sheet thickness to become almost the size of the ion gyro-radius. Their experimental analysis, however, was solely based on MHD physics and the non-MHD physics mechanisms were not discussed.

\section{Measurements in the Non-MHD Experiments}

In a linear plasma device (LPD) (Stenzel and Gekelman, 1981; Gekelman and Stenzel, 1982; Gekelman and Pfister, 1988) a reconnection region was created by driving currents 

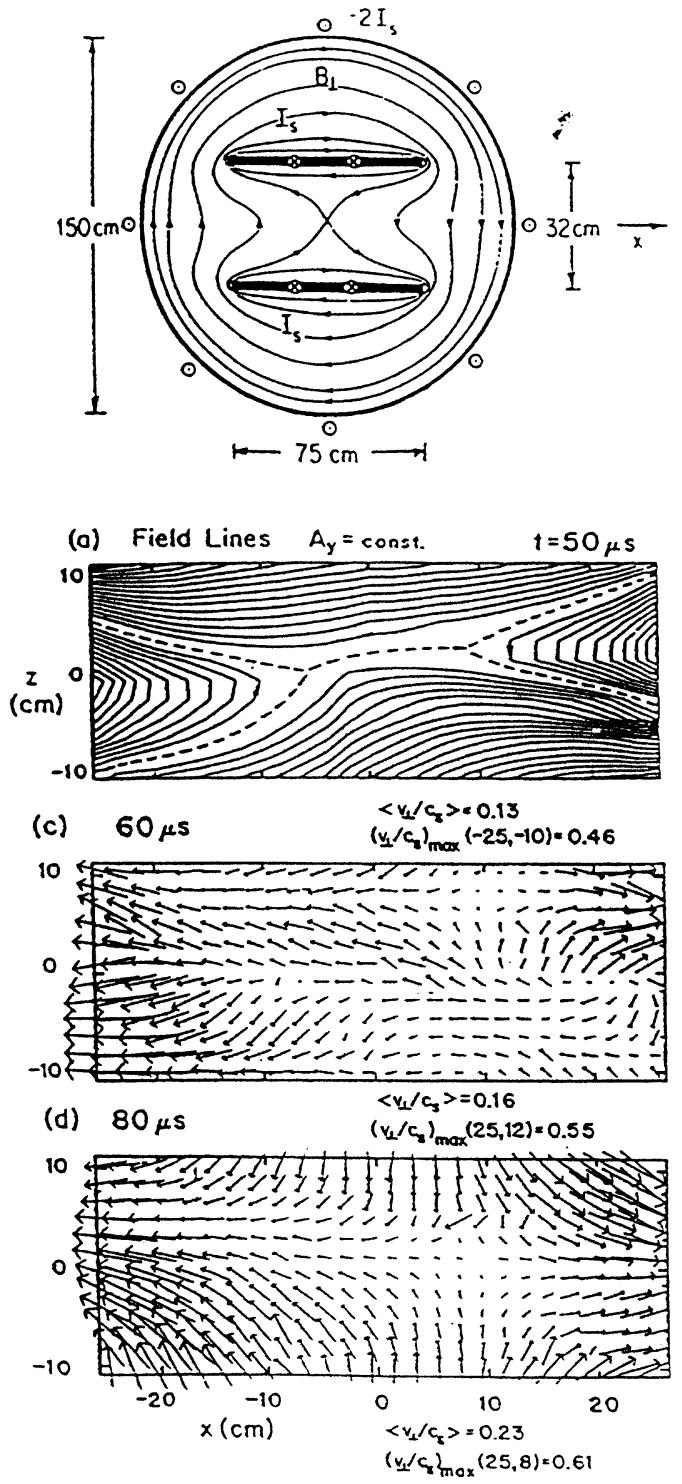

Fig. 3. Results from Stenzel and Gekelman's experiments. (a) Cross sectional view of LPD. (b) Reconnecting field line contours at $t=50$ $\mu \mathrm{sec}$. (c) and (d) Measured ion velocity vectors at an axial position $z=87 \mathrm{~cm}$ for $t=60$ and $80 \mu \mathrm{sec}$.

in the parallel sheet conductors shown in Fig. 3(a), and a detailed local study of magnetic reconnection was performed in the non-MHD regime in which ions were not magnetized. There was sizable axial magnetic field superposed on the reconnecting fields. The experiments were carried out in a cylindrical vacuum chamber (1.5 m-diameter, $2 \mathrm{~m}$ length) in which a low pressure $\left(p<10^{-4}\right.$ torr, $\left.\mathrm{Ar}, \mathrm{H}_{2}\right)$ discharge is produced with a one-meter diameter oxide coated cathode. The base plasma parameters are $n_{e} \sim 10^{12} \mathrm{~cm}^{-3}, T_{e} \sim 10$ $\mathrm{eV}$, electron mean free path $\sim 200 \mathrm{~cm}$ for electron and ion Coulomb collisions, axial magnetic field $12-100 \mathrm{G}$, and $\beta \sim$ 1 for discharges of $I p \sim 1500$ A.

This linear plasma experiment was very useful for studying non-MHD mechanisms; only electrons were magnetized $\left(\rho_{e} \ll L \ll \rho_{i}\right)$ and this regime is called the electron MHD (E-MHD) regime. Detailed measurements were made to identify local microscopic physics issues associated with neutral sheet formation. In particular ion flows were monitored and the characteristics of turbulence were measured. A neutral current sheet was seen to develop in less than two Alfven transit times $\left(\tau_{A} \sim 20 \mu \mathrm{sec}\right)$. The neutral sheet became narrower as it was measured further from the cathode. Figure 3(b) shows field lines through constant vector potential Ay at $y=137 \mathrm{~cm}$ from the cathode and at $t=50 \mu \mathrm{sec}$; in their experiment $y$ denotes the axial distance from the cathode and $t=0$ is the start time of the discharge. After a few Alfven times, the plasma was observed to develop the classic flow pattern, jetting from the neutral sheet with velocities close to the Alfven speed. In particular, the typical 2-D features of particle acceleration were verified (Gekelman and Stenzel, 1982). Figures 3(c) and (d) depict typical 2-D ion flows drifting from diffusion region to outside in perpendicular to the neutral line at $t=60$ and $80 \mu \mathrm{sec}$. It is important to note that this classical MHD flow pattern is generated by unmagnetized ions. It should be noted that the magnetized electrons are expected to experience the $j \times b$ force and the ions are coupled to the electrons via space charge forces. Spatial and temporal measurements of plasma pressure nkTe, magnetic force density $(j \times B)$ and ion velocities were performed.

Among the numerous observations from this experiments, the following results were concluded to be important: (1) formation of a long flat neutral sheet stable against tearing modes; (2) buildup of hot dense plasma region near the edge of the neutral sheet; (3) anomalous scattering of ions by waves during the reconnection process; and (4) anomalous resistivity deduced from generalized Ohm's law with all fields and currents measured. Figure 4 shows spatial profiles
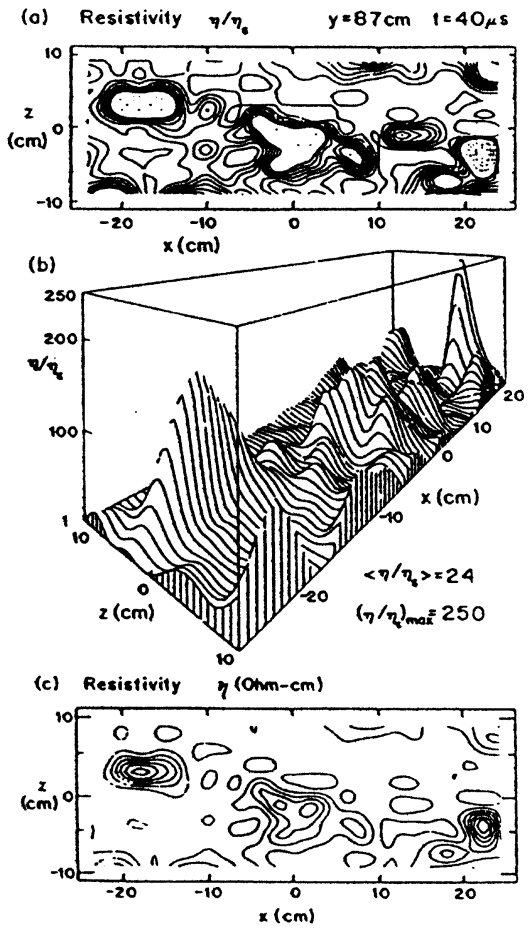

Fig. 4. Measured resistivity, both absolute and normalized to the Spizer resistivity at a specific time. (a) Contours of nomalized resistivity. Contour spacing $=20$. (b) 3-D presentation of normalized resistivity. (c) Contours of absolute resistivity, $\eta_{\max }=1.67 \Omega-\mathrm{cm}$. $D=0.25 \Omega-\mathrm{cm}$. 


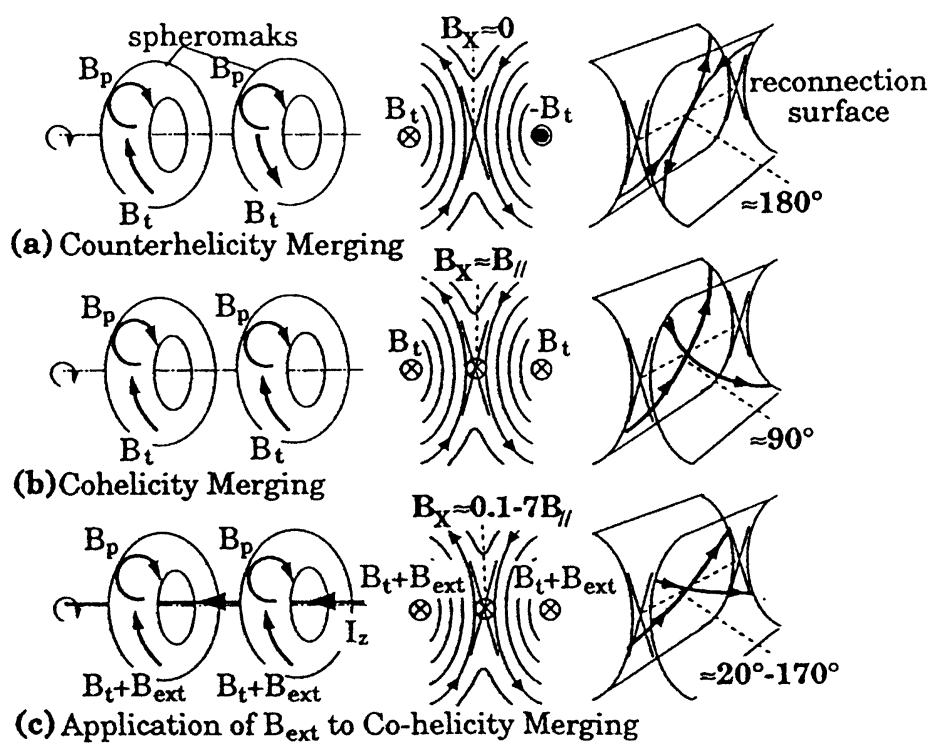

Fig. 5. Structures of reconnection region with bias magnetic field $B_{x}$. (a) Counter-helicity merging, (b) and (c) co-helicity merging with and without external toroidal field.

of measured resistivity together with the normalized one by the classical resistivity. The resistivity was often found to be peaked at the edge of the neutral sheet. The ion waves in the neutral sheet region were identified as ion acoustic waves and/or the oblique whistler waves. The polarization and propagation characteristics of these waves were measured in detail. It was concluded that the observed anomalous resistivity was in large part due to ion acoustic turbulence, although higher frequency waves were present. But it was not determined whether the whistlers were responsible for the observed large ion scattering rate or the enhanced resistivity.

It was found that the evolution of the neutral current sheet depended on the strength of the axial magnetic field superposed on the plasma as well. While a classic double-Y shaped neutral sheet was found for low axial fields of less than 40 Gauss, the development of the $\mathrm{O}$-shaped magnetic island was observed when the axial field was raised substantially to 100 gauss, much larger than reconnecting field values (co-helicity reconnection). The stability of the current sheet was also investigated: when the current density in the center of the sheet exceeded a critical value, spontaneous local current disruptions were observed. This experiment was later extended to a 3-D study (Gekelman and Pfister, 1988), in which tearing of the current sheet was observed.

The LPD experiment was instructive to study the turbulent features of non-MHD reconnection region and in particular to identify the characteristics of the wave turbulence in the neutral sheet. However, one of the most important questions on reconnection, how the diffusive neutral sheet is related to the reconnection characteristic of an MHD plasma, was not answered because the conditions for an MHD plasma were not globally satisfied $\left(L<\rho_{i}, 1<S<10\right)$. For example the Harris sheet profiles, which would be expected from the pressure balance of slowly reconnecting MHD plasma, were not supported by measurements of the neutral sheet. This may suggest that the plasma was not in a global MHD equilibrium and the non-MHD phenomena observed in the diffusion re- gion could be related only partially to the reconnection of magnetic field lines. Besides, the plasma properties seemed to be influenced by the end effects: the observed positively peaked plasma potential profile at the neutral sheet appears to be determined by the supply of electrons from the end plates. This research nonetheless made a significant contribution to the understanding of the physics of plasma turbulence in the neutral sheet.

\section{Results from Plasma Merging Experiment}

In the TS-3 device at the Univ. Tokyo, a controlled reconnection experiment was carried out in MHD plasmas where the Lundquist number exceeds 100 and both electrons and ions are magnetized. Three vector component effects of magnetic reconnection were investigated by use of axially merging two toroidal plasmas (Yamada et al., 1990; Ono et al., 1993). In the TS-3 experiments, two spheromak-type plasma toroids merged together by controlling external coil currents. The two spheromaks, which were generated with opposite helicities, carry identical toroidal current with the same or the opposite toroidal field. They are called co-helicity merging or counter-helicity merging, respectively. Figure 5(a) shows the set up of the experiment in which two spheromaks of toroidal shape are created and allowed to merge together. To document the internal magnetic structure, a two-dimensional magnetic probe array is placed on an $r-z$ plane or toroidal cut-off plane as shown in the figure. Plasma parameters are; $B \sim 0.5-1.0 \mathrm{kG}, T_{e} \sim 10 \mathrm{eV}$, and $n_{e} \sim 2-5 \times 10^{14} \mathrm{~cm}^{-3}$.

As shown in Fig. 5(a) anti-parallel reconnection is realized by the counter-helicity merging of two spheromaks with equal but opposing toroidal field $B_{t}$. The merging angle of reconnecting field lines is always 180 degree as shown in Fig. 5(a). As shown in Figs. 5(b) and (c), reconnections with oblique angle occur with and without an external field being superposed to the co-helicity merging with parallel internal toroidal fields. Figure 6 presents measured profiles of the orientation, $\theta$, of merging field lines around the dif- 

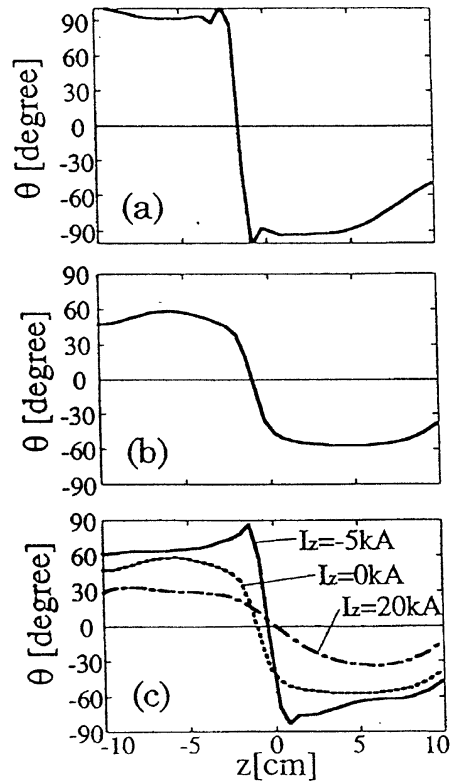

Fig. 6. Profile of merging angle of reconnecting field lines for (a) counter-helicity merging spheromaks, (b) co-helicity merging spheromaks, and (c) co-helicity t merging of spheromaks with external toroidal field.

fusion region. Counter-helicity merging generates a sharp neutral sheet while co-helicity merging with additional external toroidal field creates a much broader neutral sheet. The thickness of this neutral sheet was found to strongly depend on the merging angle as well as the external driving force. In a strongly driven case, the thickness was found to be shorter than the ion gyro-radius (Ono et al., 1997) for a short period. It was found that counter-helicity merging proceeds much faster than the co-helicity. Violent plasma acceleration was observed as a sling shot effect in the toroidal direction when field lines contracted after the merging of two toroidal plasmas of the opposite helicities (Yamada et al., 1990). This acceleration mechanism and direction is different from that conjectured in the typical 2-D models. The Doppler shift of $H_{\beta}$ and CII lines were monitored to measure radial profile of plasma acceleration and $T_{i}$. The measured radial profiles of the global plasma (ion) velocity in the toroidal direction demonstrated a sling shot type shear acceleration (Ono et $a l ., 1996)$. Strong ion heating up to $T_{i}=200 \mathrm{eV}$ was measured during reconnection. This observed strong ion heating and the 3-D sling shot acceleration are clear demonstration of conversion of magnetic energy to plasma kinetic energy. The SSX group (Kornack et al., 1998) recently obtained a similar result by reporting that 'the super Alfvenic ion flow appears to be predominantly in the plane containing reconnecting field'. In TS-3, the global characteristics of merging plasmas are primarily being studied, generating many significant findings on merging rate, ion heating and plasma acceleration.

\section{MRX (Magnetic Reconnection Experiment) Re- search}

The MRX device was built to investigate the fundamental physics of magnetic reconnection in MHD plasmas (Yamada et al., 1997a, b). On MRX the local features of the reconnection layer have been extensively studied while both the local and global physics issues and their interrelationship continue to be addressed. The overall initial geometry is axisymmetric (and hence two-dimensional), but can be made nonaxisymmetric to study 3 -D characteristics of merging. These plasmas have a high conductivity $\left(S \sim 10^{3}\right)$ with the ion gyro-radius being much smaller than the plasma size.

The first goal of this experiment was to measure the precise local features of the reconnection layer. Experiments have been carried out in the double annular plasma set-up in which two toroidal plasmas with annular cross section are formed independently around two flux cores and magnetic reconnection is driven in the quadrupole field shown in Fig. 7. Each flux core (Darkened section in Fig. 7) contains a toroidal field (TF) coil and a poloidal field (PF) coil. At first a quadrupole poloidal magnetic field is established by the PF coil currents(which flow in the toroidal direction), plasma discharges are created around each flux core by induction of pulsing currents in the TF coils (Yamada et al., 1997b). After the annular plasmas are created, the PF coil current can be increased or decreased. In the case of rising PF current, the poloidal flux in each plasma increases and is "pushed" toward the x-point (push mode). In the case of decreasing PF current, the poloidal flux in the common plasma is "pulled"

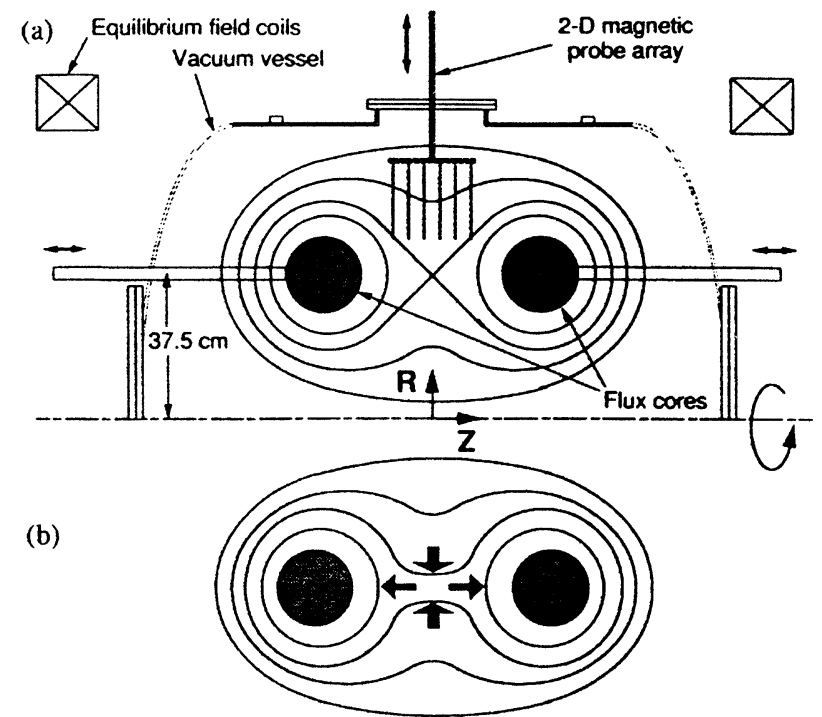

(c)

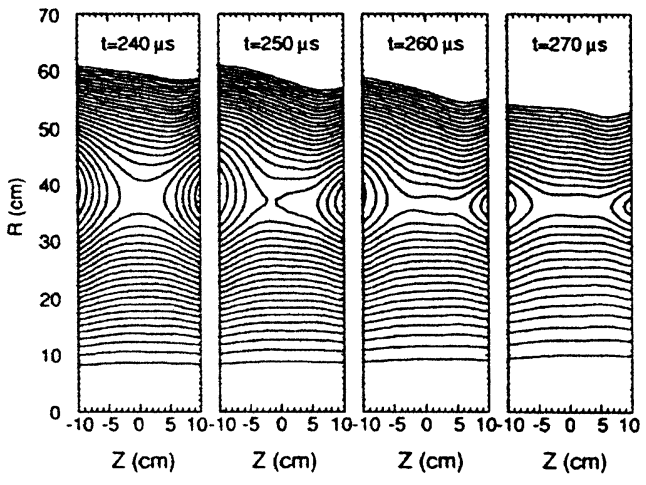

Fig. 7. Experimental setup for MRX plasma, reconnection sequence b) and evolution of measured flux contours. 


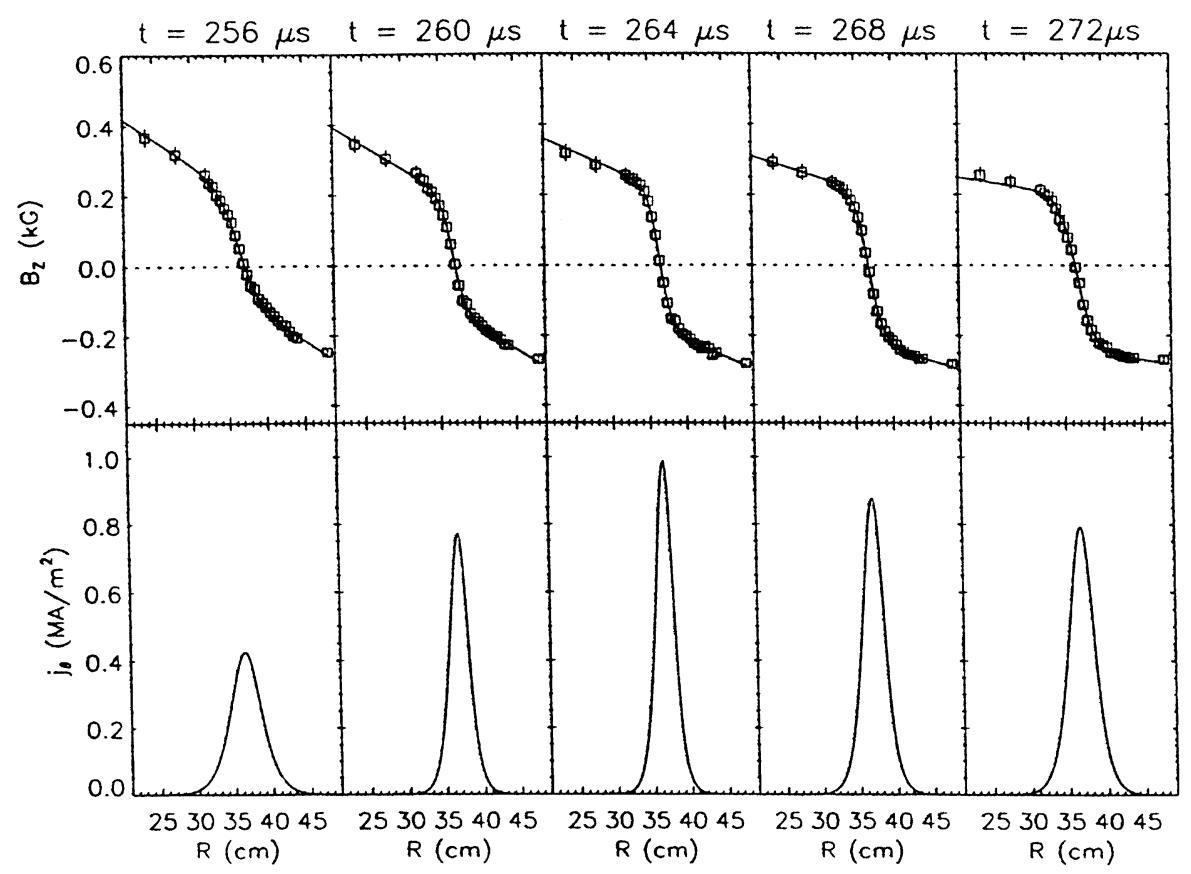

Fig. 8. Time evolution of radial profile of reconnecting field (top) and neutral sheet current taken in a single discharge. $B_{z}$ are fit to the Harris profile with a background quadrupole profile superposed.

back toward the $\mathrm{x}$-point (pull mode). For standard conditions $\left(n_{e} \sim 0.1-1 \times 10^{14} \mathrm{~cm}^{-3}, T_{e} \sim 10-20 \mathrm{eV}, B \sim 0.5-1.0 \mathrm{kG}\right.$, $S \sim 500$ ), MRX creates strongly magnetized MHD plasmas. Both the electron and ion gyro-frequencies are above the respective collision frequencies $\left(\omega_{c e} \tau_{e}>400, \omega_{c i} \tau_{e} \sim 10\right)$ for typical Hydrogen discharges of 3-6 mtorr. The mean-freepath for electron-ion Coulomb collisions is approximately $2-10 \mathrm{~cm}$. To measure the internal magnetic structure of the reconnection on a single shot, a two-dimensional magnetic probe array is placed in the $R-z$ plane or toroidal cut-off plane, as shown in Fig. 7; $z$ is in the axis of the vacuum vessel. The profile of the neutral sheet was carefully measured and two distinctively different shapes of neutral sheet current layers were identified, depending on the third [axial] vector component of the reconnecting magnetic fields. When no magnetic reconnection is induced, a typical X-shape separatrix region is observed. As poloidal flux is driven toward the diffusion region, a neutral sheet is formed. Without the third component (null-helicity reconnection), a thin double$\mathrm{Y}$ shaped diffusion region is clearly identified as shown in Fig. 7(c). With a significant third component (co-helicity reconnection), an O-shaped sheet current appears (Yamada et al., 1997a). The neutral current sheet in the null-helicity merging case is much narrower than in the co-helicity merging case.

It is found that reconnection of null-helicity plasmas occurs much faster than that of co-helicity plasmas, which confirms the earlier data obtained in the merging experiments on TS-3 (Yamada et al., 1990; Ono et al., 1993). A cause of the observed slower reconnection for co-helicity merging has been attributed to the effects of toroidal magnetic field pressure which generate a strong back pressure at the down stream side of reconnection channel.

The current density obtained from magnetic probe data provides the profile of the neutral sheet current for a given sequence of shots. A nearly symmetric profile of the neutral sheet current was observed during null-helicity reconnection. The width of the neutral sheet during null-helicity reconnection is always much narrower than the width during co-helicity merging. It is natural to question whether the welknown Harris equilibrium, which was derived as a 1-D equilibrium between a confining magnetic field and plasma pressure, could be observed and whether the magnetic reconnection process would affect the neutral sheet profiles given in the Harris equilibrium. Precise magnetic field profiles of the MRX neutral sheet have been measured by a highresolution 1-D magnetic probe array, which contains magnetic pickup coils every $5 \mathrm{~mm}\left(\sim 1 / 4\left\langle\rho_{i}\right\rangle\right)$ and is inserted across the neutral sheet.

The evolution of the reconnecting field component $B_{z}$ gives the radial profile evolution of the neutral sheet current based on the relationship of $j_{t}=\operatorname{Curl} B$. Figure 8 presents the radial profiles of reconnecting field $B_{z}$ and deduced current density profile $j_{t}(r)$ together with fitted Harris profiles.

$$
B_{Z}(R)=-B_{0} \tanh ^{2}\left[\left(R-R_{0}\right) / \delta\right]+b_{1} R+b_{2},
$$

from which the current density $j_{t}$ is derived,

$$
j_{t}(R)=\frac{B_{0}}{\mu_{0} \delta} \operatorname{sech}^{2}\left[\left(R-R_{0}\right) / \delta\right] .
$$

The current sheet thickness $\delta$ is given by

$$
\delta=\frac{c}{\omega_{p i}} \frac{\sqrt{2\left(T_{e}+T_{i}\right) / m_{i}}}{V_{i}-V_{e}}=\frac{c}{\omega_{p i}} \frac{\sqrt{2} V_{s}}{V_{\mathrm{drift}}},
$$

where $V_{s} \equiv \sqrt{\left(T_{e}+T_{i}\right) / m_{i}}$ and $V_{\mathrm{drift}} \equiv V_{i}-V_{e}$ is the relative drift between ions and electrons. The factors $b_{1}$ and $b_{2}$ 
are determined by background quadrupole and equilibrium fields. It was shown that the 1-D Harris solution satisfies MHD force balance, $j \times B=-d p / d r$. Radial profiles of magnetic field and plasma pressure in MRX have been measured to verify this force balance.

It is found that the static force balance is maintained between incoming magnetic field and the neutral sheet plasma pressure during the quasi-steady state phase of reconnection. Deviation from pressure balance occurs in the beginning and ending phases without changing the magnetic profile shape from that of the Harris solution.

The neutral sheet thickness $\delta$ was experimentally determined accurately from the fit and compared with Eq. (7). By varying the discharge voltage and using both hydrogen and deuterium fill gas (to change the density and mass, respectively), a range of $\delta$ and $c / \omega_{p i}$ values were measured experimentally and an excellent agreement with a generalized Harris theory was found (Yamada et al., 2000) as shown. Figure 9 shows that the data match well with $\delta \sim 0.35\left(c / \omega_{p i}\right)$, the data scales with $c / \omega_{p i}$. Since 1996, it has been repeatedly observed on MRX (Yamada et al., 1997a, b) and other devices (Kornack et al., 1998; Ono et al., 1997) that the neutral sheet thickness scales roughly in equal with the ion gyro-radius as well as with the ion skin depth. This is the most detailed and conclusive analysis so far on the profile of the neutral sheet.

The excellent fit of MRX magnetic data points to the Harris profile and the agreement between measured and predicted thickness are remarkable since the MRX plasma is undergoing reconnection while Harris did not take into account reconnection. However, based on the discussions in the recent paper (Yamada et al., 2000) regarding the effects of dissipation, the excellent agreement indicates that the Harris profile is the most natural one for a quasi-steady-state re-

\section{(a)}
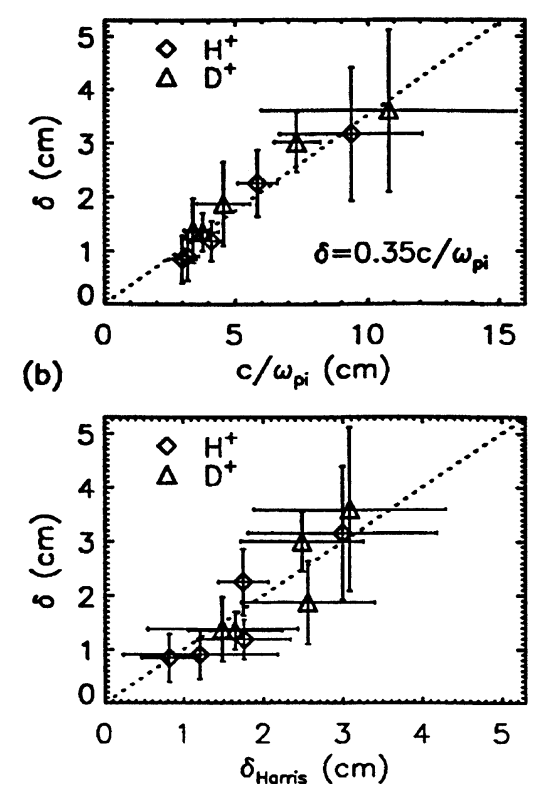

Fig. 9. Measured neutral sheet thickness vs (a) ion skin depth $c / \omega_{p i}$ and (b) Harris value given by Eq. (7). connecting plasma sheet. Even in the collisionfree regime, fluctuations are expected to create frictional forces between particles and one expects that the quasi-static equilibrium can be maintained during the reconnection process.

One of the most important local physics issues for magnetic reconnection is how magnetic energy is converted into plasma thermal energy. It was found that a significant amount of magnetic energy is converted to thermal energy of the neutral sheet plasma during reconnection and that the energy conversion rate is much larger than the value expected from classical dissipation mechanisms. Recently, local ion heating due to reconnection has been measured (Hsu et al., 1999) in MRX using a novel Ion Dynamic spectroscopy probe placed inside the neutral sheet. The results are reported in more detail by another paper in this conference ( $\mathrm{Ji}$ et al., 2000). The ion heating rate was found to be much larger than the values predicted by classical dissipation. Recently built Swarthmore Spheromak Experiment (SSX) was also utilized to study magnetic reconnection (Kornack et al., 1998). While their results are consistent with the results from TS-3 and MRX, a burst of plasma flow at the Alfven speed was observed in the reconnection plane.

Another important finding to date is that the enhancement factor of the measured resistivity over its classical values (or "the anomaly factor") is strongly dependent on the collisionality (Ji et al., 1998). In the collisional regime where the mean-free-path is comparable to the current sheet thickness, the anomaly factor is near unity, i.e., almost no resistivity enhancement is found. However, in the collisionless regime where the mean-free-path is much longer than current sheet thickness, the anomaly factor is increased to the order of 10. In earlier studies, it was found that a generalized Sweet-Parker model, which incorporates compressibility, downstream pressure and the effective resistivity, can explain the reconnection rate in MRX (Ji et al., 1998; Ji et al., 1999). In the MRX plasma, the lower hybrid waves are expected to play an important role in determining friction of electron flow against ions, a definitive study is needed to find a relationship between the observed enhanced resistivity and the amplitude of specific waves.

A significant implication of the MRX results to date is that a stable 2-D reconnection neutral sheet with axisymmetric geometry is consistent with both the Sweet-Parker and the Harris models with generalizations. The relationship $\delta \sim$ $c / \omega_{p i}$ also suggests that the electron drift velocity (relative to ions) is limited to a value equal to a constant on the order of 3 or 4 times the ion thermal speed (Ji et al., 1999). These results have led us to suspect that a current-driven instability might be excited to limit the current. The present efforts on MRX are devoted to identifying the cause of the enhanced resistivity, including macro- and micro-instabilities.

\section{Progress in Measuring Turbulence in the Neu- tral Sheet}

In searching for physical mechanisms responsible for the observed fast reconnection or "anomalous resistivity" in MRX, electrostatic and electromagnetic high-frequency fluctuations have been just recently identified and measured. This measurement was made possible by technical advancements in two areas: (1) boost of signal currents by a miniature 


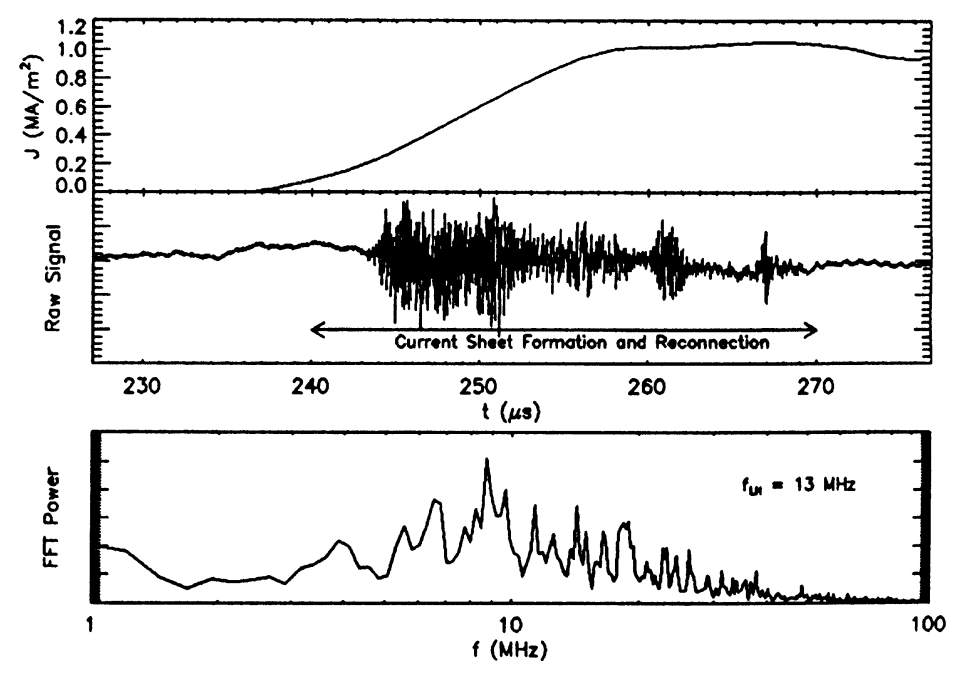

Fig. 10. Time evolution of neutral sheet current, fluctuation signal at the neutral sheet and fluctuation spectrum. The waves are identified as lower hybrid drift waves.

transducer installed in the probe shaft right after the probe tips, and (2) reduction of high-frequency background noises (by a factor of about five) by proper impedance matching of power cables from the capacitor banks to the MRX flux core coils. Initial data indicate that electrostatic fluctuations peak at the edge of current sheet while the electromagnetic fluctuations peak at the center of current sheet. The measured frequency spectra also show that most fluctuations are in the frequency range between the ion cyclotron and lower hybrid frequencies, qualitatively consistent with our theoretical model (Carter et al., 2000). Figure 10 shows the time evolution of the neutral sheet current and the fluctuation signal picked up by a double Langmuir probe immersed in the neutral sheet together with the frequency spectrum. In the most recent campaign of MRX experiments, detailed characteristics of the electro-static turbulence, dispersion relations, spatial profile, frequency spectrum etc., have been documented (Ji et al., 2001). The dispersion relations of the fluctuations have been determined by measuring their wave number spectra and phase velocities. The waves agree with the propagation characteristics of the lower hybrid drift waves. The immediate next step is to measure correlations of these fluctuations with the observed resistivity enhancement and ion heating.

\section{Summary Discussions}

We have reviewed the recent series of magnetic reconnection experiments carried out in the past two decades putting a special focus on the profile of the neutral sheet or diffusion region. Recently it has been clearly recognized that the neutral sheet thickness is often of the order of the ion gyro-radius and the standard one-fluid MHD theory does not properly describe the physics properties of the sheet.

In the modern experiments, the familiar 2-D feature, a double-Y shaped diffusion region, was identified when there is no or very little axial magnetic field (third vector component). In co-helicity merging with a sizable third field component, an O-shaped diffusion region often appears and the reconnection rate decreases substantially, which is at- tributed to the axial field pressure and the incompressibility of plasma. When the axial field component is near zero (the reconnecting angle is near 180 degrees), the reconnection speed is maximized. This observation is consistent with the well known observation in the dayside magnetosphere in which southward (antiparallel) interplanetary wind (IMF) reconnects much faster with the earth dipole field than northward IMF $\left(0^{\circ}-45^{\circ}\right.$ merging angle $)$.

Inside the current sheet layer, the standard one-fluid MHD formulation breaks down. Syrovatskii et al. (1981) realized that the thickness of the sheet appeared to be determined by the pressure balance of the reconnecting magnetic field and the plasma kinetic pressure which makes the sheet thickness almost the size of ion gyro-radius. Their experimental analysis, however, was solely based on MHD physics and the implication of the sheet thickness for non-MHD physics mechanisms was not discussed. The LPD experiment made significant measurements of the characteristics of non-MHD turbulence in the diffusion region. There were many important findings including: (1) observation of long flat neutral sheets which were stable against tearing modes; (2) ions scattered off waves during the reconnection process; and (3) anomalous resistivity which was deduced from the generalized Ohm's law when all fields and currents were measured. However, an important question, how the diffusive neutral sheet is formed in an MHD equilibrium plasma, was not addressed because the conditions for an MHD plasma were not satisfied globally. The Harris equilibrium was not observed in the current sheet of LPD. The TS-3 and MRX reconnection experiments were carried out in plasmas which globally satisfy the MHD criteria where the Lunquist numbers exceeds 100 with both magnetized electrons and ions $\left(\rho_{e} \ll \rho_{i} \ll L\right)$. And the thickness of this thin current layer is found to be on the order of the ion gyro-radius in these devices.

The neutral sheet profile during magnetic reconnection has been measured precisely in MRX. The magnetic field profile is seen to agree remarkably well with the Harris sheet profile even though the Harris theory does not take into account 
reconnection and associated dissipation. The measured neutral sheet thickness is seen to scale with the ion skin depth, $c / \omega_{p i}$ over a wide range of discharge conditions. These results imply that one can include the effects of quasi-steady state reconnection within the basic formulation of the Harris sheet. Because the Harris-theory's shifted Maxwellian velocity distribution function satisfies the full Fokker-Planck equation, it appears to be the natural state for a reconnecting plasma.

In the case of anti-parallel reconnection (null-helicity case), the ion gyro-radius is on the order of the ion skin depth, which indicates that the magnetic pressure in the upstream is balancing with the plasma kinetic pressure at the reconnection region, since $B^{2} / 2 \mu_{0} \sim n T_{e}+n T_{i}$ would lead to $c / \omega_{p i} \sim \rho_{i}$. This result is in very good agreement with numerical simulations carried out independently by Drake et al. (1997) and Horiuchi and Sato (1997) as well as with space observations. Both in the geotail region and the magnetopause, it has been often observed that the neutral thickness is of the order of the ion gyro-radius ( $\sim$ a few hundred $\mathrm{km}$ ) (Kivelson and Russell, 1995).

Collisionless reconnection (where the collision mean-freepath is much longer than the scale length of the neutral sheet or wave lengths of turbulence) will be the subject of intensive investigations in both laboratory experiments and numerical simulations in the future. It should be noted that the physics of collisionless reconnection has great relevance to solar flare and magnetospheric phenomena, which occur mostly in the collisionless regime. In MRX experiments with collisional conditions where the mean-free-path is comparable to the current sheet thickness, the reconnection resistivity is found to be classical, i.e., almost no resistivity enhancement is found. However, in the collisionless regime where the mean-free-path is much longer than the current sheet thickness and the wavelengths, the resistivity enhancement factor is increased to the order of 10. An enhancement factor of more than 100 was measured in the LPD device. Although this is suspected to be due to wave-particle interactions, conclusive work is yet to be carried out. In the electron MHD regime in the LPD experiments whistler waves were suspected to be the cause of the observed anomalous resistivity but it was concluded that the enhanced resistivity was in large part due to the current driven ion acoustic turbulence in the neutral sheet and the role of whistler waves on the anomalous resistivity was not verified in LPD. A new experiments have been initiated at MIT to address the physics magnetic reconnection in a collisionless regime (Egedal and Fasoli, 2001).

In searching for physical mechanisms responsible for the observed fast reconnection or "enhanced resistivity" in MRX, electrostatic and electromagnetic high-frequency fluctuations have been just recently identified and their popagation chracteristics have been measured. Initial data indicate that electrostatic fluctuations peak at the edge of current sheet while the electromagnetic fluctuations peak at the center of current sheet. The measured frequency spectra also show that most fluctuations are in the frequency range between the ion cyclotron and lower hybrid frequencies. The measured wavelengths are found to be smaller than the collision meanfree-path lengths in the low density regime. Experiments are being carried out to measure the correlation of these fluctuations with the observed resistivity enhancement and ion heating, which is crucial to understand the mechanisms of fast collisionless reconnection.

Acknowledgments. The author appreciates many useful inputs from Russell Kulsrud, Hantao Ji, Troy Carter and MRX staff. Discussions with Reiner Stenzel and Yasushi Ono were very valuable. Work jointly supported by DoE, NASA, and NSF.

\section{References}

Baum, P. J. and A. Bratenahl, Magnetic reconnection experiments, Adv. Electron Phys., 54, 1-67, 1980, reference there in.

Biskamp, D. E., Schwarz, and J. F. Drake, Ion controlled magnetic reconnection, Phys. Rev. Lett., 75, 3850-3853, 1995.

Brown, M. R., Experimental studies of Magnetic reconnection, Phys. Plasmas, 6, 1717-1724, 1999.

Carter, T. et al., AGU 2000 Spring Meeting EOS, Supplement, S360, 2000.

Drake, J. F., R. G. Kleva, and M. E. Mandt, Structure of thin current layers: Implication for magnetic reconnection, Phys. Rev. Lett., 73, 1251-1254, 1994.

Drake, J. F. et al., Geophys. Res. Lett., 24, 2921-2924, 1997.

Egedal, J. and A. Fasoli, to be published, 2001.

Frank, A. G., Proc. Lebedev Phys. Inst. 74: 108, 1974.

Gekelman, W. and R. L. Stenzel, Magnetic field line reconnection experiments, J. Geophys. Res., 86, 659-666, 1981, JGR, 87, 101-110, 1982, $J G R, \mathbf{8 9}, 2715-2733,1984$.

Gekelman, W. and H. Pfister, Experimental observations of the tearing of an electron current sheet, Phys. Fluids, 31, 2017, 1988.

Harris, E. G., Nuovo Cimento, 23, 115, 1962.

Horiuch, H. and T. Sato, Particle simulation study of collisionless driven reconnection in a sheared magnetic field, Phys. Plasmas, 4, 277, 1997.

Hsu, S. et al., Local measurement of non-classical ion heating during magnetic reconnection, Phys. Rev. Lett., 84, 3859-3862, 2000.

Ji, H. et al., Experimental test of the Sweet-Parker model of magnetic reconnection, Phys. Rev. Lett., 80, 3256-3259, 1998.

$\mathrm{Ji}$, H. et al., Magnetic reconnection with Sweet-Parker characteristics in 2-D laboratory plasmas, Phys. Plasmas, 6, 1743-1749, 1999.

Ji, H., T. Carter, S. Hsu, and M. Yamada, Study of local reconnection physics in a laboratory plasma, Earth Planets Space, 53, this issue, 539-545, 2001

Kivelson, G. and C. T. Russell, Introduction to Space Physics, Cambridge University Press, London, 1995.

Kornack, T. W. et al., Experimental observation of correlated magnetic reconnection and Alfvenic ion jet, Phys. Rev. E, 58, R36-R39, 1998.

Kulsrud, R., Magnetic reconnection in an MHD plasma, Phys. Plasmas, 5, 1599-1606, 1998.

Ohyabu, N. et al., Strong ion heating in a magnetic neutral point discharge, Phys. Fluids, 17, 2009-2013, 1974.

Ono, Y. et al., Experimental investigation of three-dimensional magnetic reconnection by use of two colliding spheromaks, Phys. Fluids B, 5, 3691-3701, 1993

Ono, Y. et al., Experimental investigation of three-component magnetic reconnection by use of merging spheromaks and tokamaks, Phys. Plasmas, 4, 1953-1963, 1997.

Ono, Y. et al., Ion acceleration and direct ion heating in three component magnetic reconnection, Phys. Rev. Lett., 76, 3328-3331, 1996.

Parker, E. N., Sweet's mechanism for merging magnetic fields in conducting fluids, J. Geophys. Res., 62, 509, 1957.

Parker, E. N., Cosmical Magnetic Fields, Oxford, Claredon Press, 1979.

Parker, E. N., The reconnection rate of magnetic fields, Astrophys. J., 180, 247-252, 1973.

Petschek, H. E., Magnetic field annihilation, NASA Spec. Pub. SP-50, 425, 1964

Priest, E. R. and T. Forbes, Magnetic reconnection, Cambridge Univ. Press, Cambridge, U.K., 2000.

Sato, T. and T. Hayashi, Externally driven magnetic reconnection and powerful magnetic energy converter, Phys. Fluids, 22, 1189-1202, 1979.

Shay, M. A. et al., Structure of dissipation region during magnetic reconnection, J. Geophys. Res., 103, 9165-9176, 1998.

Stenzel, R. L. and W. Gekelman, Magnetic field line reconnection experiments I. Field topologies, J. Geophys. Res., 86, 649-658, 1981.

Stenzel, R. L. et al., Magnetic field line reconnection experiments 4, $J$. Geophys. Res., 87, 111-117, 1982. 
Stenzel, R. L., W. Gekelman, and N. Wild, Magnetic field line reconnection experiments 5. Current disruptions and double layers, J. Geophys. Res., 88, 4793-4804, 1983.

Sweet, P. A., The neutral point theory of solar flares, in Electromagnetic Phenomena in Cosmical Physics, edited by B. Lehnert, p. 123, Cambridge Press, New York, 1958.

Syrovatskii, S. I. et al., Current distribution near the null line of magnetic field and turbulent plasma resistance, Sov. Phys. Tech. Phys. Engl. Tran., 18, 580, 1973.

Syrovatskii, S. I., Pinch sheets and reconnection in astrophysics, Ann. Rev. Astron. Astrophys., 19, 163, 1981, and references there in.

Yamada, M. et al., Magnetic reconnection of plasma toroids with co- and counter-helicity, Phys. Rev. Lett., 65, 721-724, 1990.

Yamada, M. et al., Identification of Y-shaped and O-shaped diffusion regions during magnetic reconnection in a laboratory plasma, Phys. Rev. Lett., 78, 3117-3120, 1997a.

Yamada, M. et al., Study of driven magnetic reconnection in a laboratory plasma, Phys. Plasmas, 4, 1936-1944, 1997b.

Yamada, M. et al., Experimental investigation of the neutral sheet profile during magnetic reconnection, Phys. Plasmas, 7, 1781-1787, 2000.

M. Yamada (e-mail: myamada@pppl.gov) 